\title{
Sentinel Lymph Node Biopsy: Past and Present Implications for the Management of Cutaneous Melanoma with Nodal Metastasis
}

\author{
Abel Gonzalez ${ }^{1,2}$ (D) \\ Published online: 30 October 2018 \\ (c) The Author(s) 2018
}

\begin{abstract}
Although significant progress has been made in the understanding of melanoma pathophysiology and therapy, patients with metastatic melanoma still have a poor prognosis. The management of regional nodes remains a matter of debate. By replacing elective lymph node dissection, sentinel lymph node biopsy has revolutionized the treatment of malignant melanoma. In this paper, the history of the procedure is traced, and the indication for completion lymphadenectomy after positive sentinel node biopsy is discussed in light of the recent studies that addressed this issue. The role of adjuvant therapies in the management of patients with stage III melanoma is also discussed.
\end{abstract}

\section{Key Points}

Surgery is the mainstay of treatment in the early stages of malignant cutaneous melanoma.

Sentinel node biopsy, a minimally invasive surgical technique introduced in the 1990's, has profoundly transformed the method of nodal staging and melanoma treatment.

Sentinel node status has proven to be the most significant prognostic indicator in patients with localized intermediate-thickness cutaneous melanoma.

\section{Introduction}

As metastases from melanoma most frequently develop in lymph nodes, sentinel lymph node biopsy (SLNB) has emerged as a key diagnostic tool for determining whether cancer has spread beyond the primary tumor site to the

Abel Gonzalez

abelgo@gmail.com

1 Mohs Surgery Unit, Institute of Oncology Angel H. Roffo, University of Buenos Aires, Av San Martin 5481, Ciudad Autónoma de Buenos Aires, Buenos Aires, Argentina

2 Unit of Cutaneous Oncology and Mohs Surgery, Institute Alexander Fleming, Conesa 1003, Ciudad Autónoma de Buenos Aires, Buenos Aires, Argentina lymph nodes. This minimally invasive procedure has successfully replaced elective lymph node dissection (ELND) as a sensitive prognostic tool and has changed the management of primary melanoma [1]. SLNB has also provided a better understanding of the nodal metastatic process, giving rise to the concepts of sentinel lymph node (SLN) tumor burden and to the status of a non-sentinel lymph node (non-SLN).

\section{Sentinel Lymph Node Biopsy: A New Era in Cutaneous Melanoma Management}

\subsection{History of the Sentinel Lymph Node Biopsy}

Prophylactic removal of regional nodes by routine ELND was recommended for the first time by Herbert L. Snow in 1892 to halt tumor progression, regardless of the absence of palpable nodes [2]. However, the efficacy of this procedure has been long debated. Several prospective randomized controlled studies [3-6] have shown that ELND has no benefit in patients with cutaneous melanoma, but subgroup analysis in the trial conducted by Balch et al. indicated that ELND improved survival in certain subgroups, such as in patients aged 60 or younger, or with 1 to 2 -mm thick or non-ulcerated tumors [3].

The primary shortcoming of ELND is that only about $20 \%$ of patients with an intermediate-thickness primary melanoma are expected to have metastases in the regional nodes, whereas $80 \%$ of patients are exposed to the morbidity of lymphadenectomy, without actual benefit [7]. Another issue 
with ELND was to identify the basins at risk of metastasis when cutaneous melanoma developed in sites with ambiguous lymphatic drainage pathways. Donald L. Morton developed a technique called cutaneous lymphoscintigraphy to map lymphatic drainage pathways in truncal or shoulder melanomas [8]. Dr Morton explained that it was during a lymphoscintigraphy - while observing the dynamic images of a lymphatic channel draining directly to a first node, and then into the secondary nodes - that the idea of the "sentinel" node was born. The first node draining directly from the site of the primary lesion was the first at risk of being reached by cancer cells spreading from the tumor. He realized that knowing the status of this "sentinel" node made it possible to know the status of the entire regional basin. This simple observation developed into the revolutionary concept of the SLNB.

In 1992, Morton et al., published an operative procedure using this technique to select and remove SLNs and identify patients with metastatic nodes [7]. Cutaneous lymphoscintigraphy with technetium-labeled dextran was used to identify the areas of primary drainage for melanomas located in ambiguous sites. Then a blue dye was injected intradermally at the melanoma site to trace the connected lymph vessels and identify the draining sentinel node(s). The SLNs were then dissected and evaluated by routine hematoxylin eosin or immunohistochemistry to detect the presence of metastatic deposits. Following SLNB, every patient included in this study had a complete lymph node dissection (CLND) and non-SLNs were also examined for metastases to evaluate the accuracy of the procedure.

SLNs were identified in 194 (82\%) of the 237 lymphadenectomy specimens. Metastases were detected in 40 (21\%) of these specimens. In contrast, the rate of metastasis in nonSLNs from these lymphadenectomy specimens was only $1 \%$ (2/194) [7]. These results confirmed that the SLN is the initial site of regional lymphatic node metastases.

The use of radioisotopes was extended from preoperative localization of the SLN to intraoperative identification. Dr Morton reported his use of intraoperative radiolymphoscintigraphy using $99 \mathrm{mTc}$-labeled albumin before the Society of Surgical Oncology in 1994 [9]. Within a few years, the prognostic significance of SLNB had been clearly demonstrated by several studies and the American Joint Committee on Cancer incorporated the tumor status of the SLNs into its staging system for melanoma in 2001 [10].

\subsection{The Multicenter Selective Lymphadenectomy Trial-1 (MSLT-I)}

The MSLT-I was designed to determine whether SLNB could be used to identify patients with clinically occult nodal metastases and to compare immediate CLND with therapeutic lymph node dissection (TLND), when nodal recurrence was observed during observation.

The MSLT-I was a large cohort study involving 2001 patients initiated by Morton et al. in 1994 [1] (Table 1). Patients included had localized cutaneous melanomas with a Breslow thickness of $1 \mathrm{~mm}$ or more, or Clark level IV-V with any Breslow thickness, and were followed up for 10 years. Patients with intermediate-thickness melanomas, defined in this study as those with 1.2 to $3.5-\mathrm{mm}$ melanomas, constituted the primary study group. This was because pretrial statistical modeling, based on data from the prospective melanoma database of the John Wayne Cancer Institute, indicated that the timing of CLND was most likely to affect survival in this subgroup. Our discussion of MSLT-I therefore focuses on this subgroup of patients.

Patients with intermediate-thickness $(n=1347)$ primary melanomas were randomly assigned to undergo wide excision and either SLNB $(n=814)$ or regional nodal observation $(n=533)$ [1].

Among the patients in the biopsy group, metastases were identified in $16.0 \%$ of patients and nodal metastases were detected during observation in $4.8 \%$ of patients with tumor-free SLN; thus, the proportion of patients with intermediate-thickness melanomas who had nodal metastases in the biopsy group was $20.0 \%$, and the estimated cumulative incidence of nodal metastases at 10 years was $21.9 \%$. In the observation group, $17.4 \%$ of patients had nodal metastases at a median of 19.2 months, and the estimated 10-year cumulative incidence of nodal metastasis was $19.5 \%$.

Ten-year disease-free survival rates were significantly higher in the biopsy group than in the observation group, $(71.3 \pm 1.8 \%$ versus $64.7 \pm 2.3 \%$, hazard ratio [HR] for recurrence or metastasis of $0.76 ; 95 \% \mathrm{CI}, 0.62$ to $0.94 ; \mathrm{p}=0.01$ ). In contrast, 10-year melanoma-specific survival rates were similar between the two treatment groups in patients with intermediate-thickness melanomas $(81.4 \pm 1.5 \%$ versus $78.3 \pm 2.0 \%$, HR of 1.12 (95\% CI, $0.76-1.67 ; p=0.56)$. Thus, this trial did not demonstrate a statistically significant therapeutic advantage of SLNB over observation with regards to melanoma-specific survival (Table 1).

The rationale for and against SLNB can be resumed by the incubator versus marker hypotheses. These are the two possible paths of metastasis from a primary melanoma. According to the incubator hypothesis, a primary melanoma initially metastasizes via the lymphatics to the SLN. The metastatic foci may remain latent (incubate) in the SLN before spreading to distant sites. Thus, removal of the positive SLN before dissemination should prevent distant metastasis. According to the marker hypothesis, a primary melanoma metastasizes simultaneously via the lymphatic and hematogenous routes. Thus, finding a positive SLN is only a marker of a primary melanoma that can metastasize, 


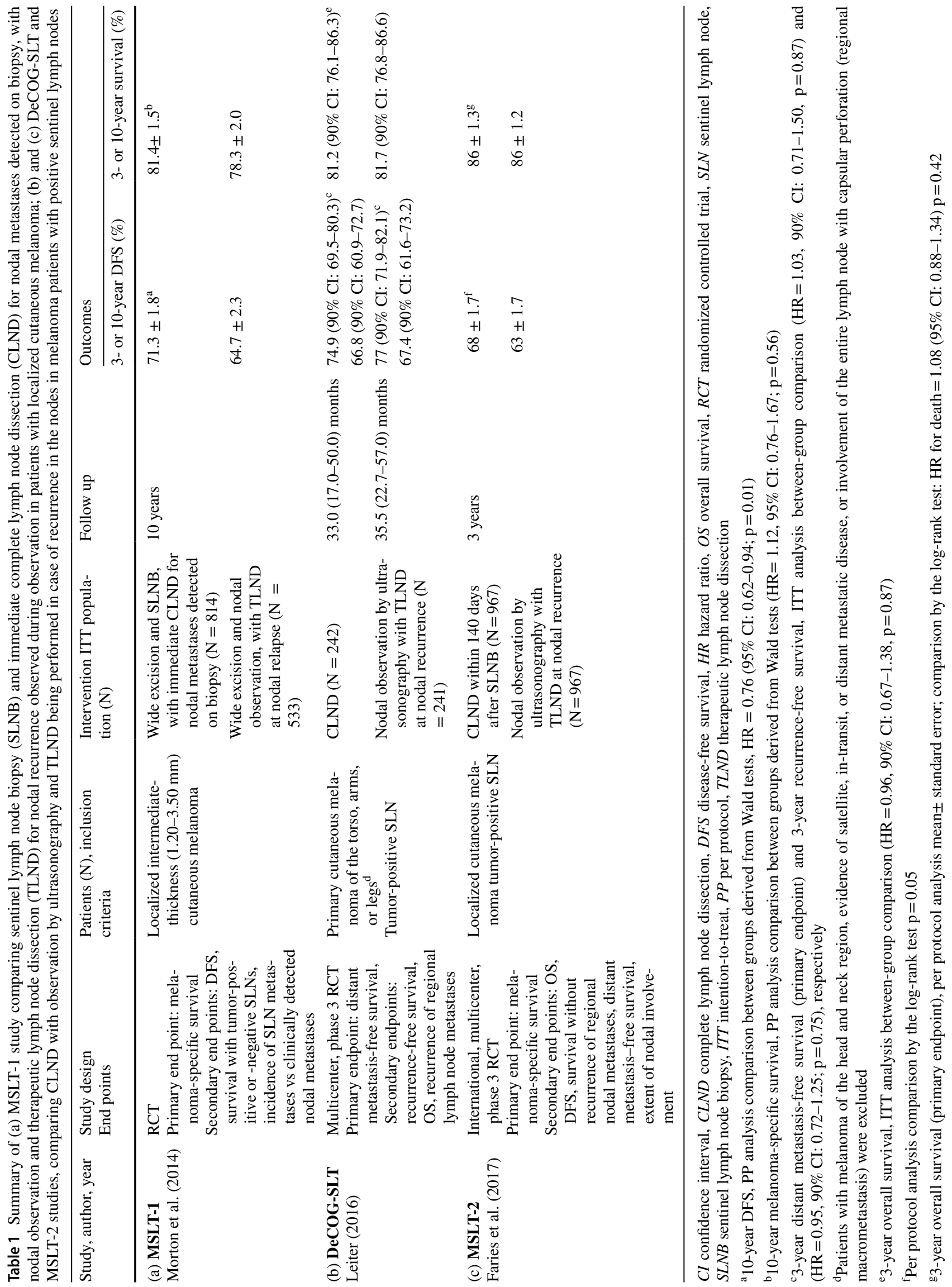


and surgical treatment of the nodes is unlikely to have any therapeutic effect on distant metastases [11].

However, the MSLT-I lacked the power to detect any potential differences in survival between patients with and without SLNB due to the dilution effect associated with only $15-20 \%$ of patients having SLN metastases and only 15-20\% of these patients having additional nodal metastases in the CLND specimen.

Although the therapeutic role of SLNB in melanomaspecific survival was not confirmed, further subgroup analyses provided important information about the prognostic value of SLN status. Firstly, analysis of the biopsy group indicated that the 10-year melanoma-specific survival rate was significantly lower among SLN-positive patients than among SLN-negative patients for intermediate-thickness melanomas $(62.1 \pm 4.8 \%$ versus $85.1 \pm 1.5 \%)$. Multivariate analysis for intermediate-thickness melanoma patients confirmed that SLN status was the most powerful prognostic factor, with about a 2.5 -fold higher risk of recurrence or death from melanoma for SLN-positive patients than for the SLN-negative patients in the biopsy group $(\mathrm{p}<0.001)$. Secondly, analysis of data from patients who developed nodal metastases indicated that early intervention for nodal disease had a significant positive impact on survival: patients with intermediate-thickness melanomas in the observation group who developed a palpable nodal recurrence and had a TLND had a significantly lower 10 -year melanoma-specific survival rate than those in the SLNB group with a positive SLN and immediate CLND (41.5\% versus $62.1 \%, p=0.006)$. Significant improvements in distant disease-free survival were also observed in patients with intermediate-thickness melanoma and nodal metastases in the biopsy group compared to those in the observation group ( $\mathrm{HR}=0.62 ; 95 \% \mathrm{CI}, 0.42-0.91$, $\mathrm{p}=0.02$ ). These results clearly indicated that the timing of the intervention for nodal disease was an important factor for reducing the risk of melanoma-related distant metastases, nodal recurrence and death in patients with intermediatethickness melanoma.

\subsection{Classification of Sentinel Node Micrometastases and Their Clinical Relevance}

Sentinel node biopsy has resulted in an unprecedented understanding of melanoma biology with, among other consequences, the emergence of the concepts of SLN tumor burden and the status of non-SLN as prognostic factors.

Three methods have been described to accurately quantify and classify micrometastases in positive SLNs and evaluate their prognostic value.

Firstly, Starz et al. described a routine S-staging micromorphometric technique using the number of 1-mm thick SLN slices with detectable tumor cells (n) and the depth of tumor cell invasion (d) as morphometric parameters [12]. On the basis of this classification, the authors analyzed the metastasisrelated mortality in 342 melanoma patients (median follow up, 36 months) in correlation with each patient's highest $\mathrm{S}$ classification. The study showed that $\mathrm{S} 1$-stage $(1 \leq \mathrm{n} \leq 2$ and $\mathrm{d} \leq 1 \mathrm{~mm}$, with clustered subcapsular metastatic deposits) and S2-stage ( $\mathrm{n}>2$ and $\mathrm{d} \leq 1 \mathrm{~mm}$, with more extended peripheral metastases) patients had a 5-year survival rate without distant metastases of over $90 \%$, similar to that of S0-stage (no detectable metastases) patients. In contrast, $\mathrm{S} 3$-stage ( $\mathrm{n}>2$ and $\mathrm{d}>1 \mathrm{~mm}$, and deeper infiltration of metastatic cells in the parenchyma) patients had a very poor prognosis with a 5-year survival rate of around $30 \%$.

To refine the selection of patients eligible for CLND, Dewar et al. established a second classification categorizing metastases based on their microanatomic distribution inside the SLN in a study involving 146 patients with SLNB-positive metastatic melanoma [13]. Using this approach, they found a significant positive correlation between the location of metastases and non-SLN involvement. None of the patients with metastatic deposits confined to the subcapsular area had involvement of the non-SLNs, whereas positive non-SLNs were detected in $11.1 \%$ of patients with combined subcapsular and parenchymal metastases, in $18.8 \%$, of patients with larger metastases confined to the paracortical area of parenchyma, in $36.8 \%$ of patients with multifocal multiple discrete deposits with some parenchymal deposits, and in $42.1 \%$ of patients with extracapsular or extensive metastases larger than $5 \mathrm{~mm}$. This study demonstrated that the location and distribution of metastatic cells within the SLN had a critical predictive value for non-SLN involvement and suggested that patients with metastases circumscribed to the subcapsular region could safely be spared complete surgical resection of the adjacent lymph nodes.

Finally, SLN tumor load can be evaluated using the very simple Rotterdam Criteria to categorize metastases based on the maximum diameter of the largest metastasis $(<0.1 \mathrm{~mm}$; $0.1-1 \mathrm{~mm} ;>1 \mathrm{~mm}$ ). Using this classification, a large multicenter study involving 388 SLN-positive melanoma patients reported that among the $10 \%$ of patients with SLN micrometastases of less $0.1 \mathrm{~mm}$, only $3 \%$ had additional positive non-SLNs [14]. The 5-year overall survival in these patients (91\%) was the same as that for patients with a negative SLN, thus these patients could be spared CLND. Therefore, SLN status is the most important prognostic factor in patients with localized melanoma and non-SLN status is the highest significant independent predictor of survival in patients with positive SLNs.

\subsection{Benefit of Complete Lymph Node Dissection in Patients with a Positive Sentinel Node Biopsy}

Immediate CLND was the established standard procedure for melanoma patients after a positive SLNB, but as 
shown in the MSLT-I trial, melanoma-specific survival was not modified by this procedure [1]. Although the eventual removal of positive non-SLNs and improved regional control are considered as potential advantages of CLND, nodal disease is limited to the SLNs in most patients and is removed by means of the SLNB. Additional positive non-SLNs are found in around 8 to $20 \%$ of patients after CLND, although some series show even higher numbers [15].

Therefore, around $80 \%$ of patients undergo surgery with all the associated risks and no actual benefit. Furthermore, follow up with ultrasound of the nodal basin may pick up recurrences at an early enough time point to allow for successful surgical treatment.

Two clinical trials were designed to clarify the therapeutic role of CLND in melanoma patients with nodal metastases by testing the hypothesis that CLND in patients with a positive SLN improved survival: the German Dermatologic Cooperative Oncology Group Selective Lymphadenectomy Trial (DeCOG-SLT) and the Second Multicenter Selective Lymphadenectomy Trial (MSLT-II) [16, 17].

The DeCOG-SLT study was a multicenter randomized phase- 3 clinical trial conducted in 41 centers across Germany on patients with melanoma of the torso, arms or legs, and a positive SLNB (Table 1). Patients were randomly assigned to either undergo CLND, or observation by ultrasonography with TLND being performed in case of recurrence in the nodes [16]. Prognostic factors were well balanced: no differences in SLN tumor burden were found between the two groups. The results showed that the number of melanoma-associated deaths was similar between patients who had CLND and those in the observation group (15\% versus 16\%). Three-year distant metastasis-free survival, overall survival, and recurrence-free survival were also similar in both groups (Table 1). Similar results were obtained after subgroup analysis by tumor load in the SLN for distant metastasis-free survival.

However, this study bore numerous limitations: head and neck patients were excluded; the study was underpowered as only 483 of the 1269 patients (34.2\%) with a positive SLNB were randomly assigned to the treatment groups due to 314 patients not meeting the inclusion criteria, 227 declining to participate and 254 having missing data; two-thirds of patients had a tumor burden below $0.1 \mathrm{~mm}$, which is less than in previous studies and has been associated with an excellent prognosis; the number of events was lower than anticipated; and finally, the median follow-up period was short (34 months). Despite these limitations, the strength of the study is bolstered by the very consistent results.

The design of the MSLT-II was very similar to that of the DeCOG-SLT, but the power of the MSLT-II was superior, with 1939 out of the 3531 patients enrolled (54.9\%) being randomly assigned to the two treatment groups, head and neck patients were included and the median follow up was
43 months (Table 1). The end results of MLST-II, published in 2017 [17], were similar to those of the DeCOG-SLT study [16]: at the 3-year follow up, the mean rate of melanomaspecific survival was almost identical between the dissection and the observation group ( $86 \pm 1.3 \%$ versus $86 \pm 1.2 \%$, respectively, $\mathrm{p}=0.4)$, whereas disease-free survival was higher in the dissection group ( $68 \pm 1.7$ versus $63 \pm 1.7 \%$; $\mathrm{p}=0.05$ ). However, CLND was found to provide improved staging and an increased rate of regional disease control. The most important disadvantage of CLND was lymphedema, which occurred in $24 \%$ of the patients in the dissection group compared with $6 \%$ in the observation group.

The MSLT-II study confirmed that the pathologic status of non-SLNs has independent prognostic value and therefore CLND provides information for risk stratification and selection of adjuvant therapy, which is lacking for patients who do not undergo CLND.

In the light of these recent studies, nodal observation appears to be a safe alternative to CLND.

However, CLND remains an available treatment option in specific populations or clinical situations which remain to be defined. Furthermore, we believe observation should be seriously discussed with patients with low adherence to follow-up visits or those who receive treatment at institutions that are not able to perform adequate nodal ultrasonography, mainly in case of high tumor burden in the SLN, or of high-risk of ulcerated or thick melanoma.

Use of CLND in head and neck melanoma patients may need to be considered separately. Firstly, data concerning this location are scarce: head and neck patients were excluded from the DeCOG-SLT study, and only $13.7 \%$ of the patients included in the MSLT-II study had head or neck melanomas. Secondly, in the MSLT-II study, the HR in the observation arm for head and neck melanoma was $1.6(p=0.07)$, compared with 1.05 for the trunk and 1 for the limbs. Finally, lymphedema-almost the only permanent complication of CLND-is not an issue in head and neck patients.

\section{Adjuvant Therapies in Resected Stage III Melanoma Patients}

Adjuvant therapy using immune checkpoint inhibitors such as ipilimumab and nivolumab, or therapies targeting melanoma-specific mutations might be recommended in melanoma patients with resected stage III positive regional lymph nodes with micrometastases, who have undergone CLND and have a high risk of subsequent relapse.

The efficacy of ipilimumab was compared to placebo treatment in a large international randomized, doubleblind, phase 3 clinical trial involving patients with stage III melanoma ( $\mathrm{N}=951$, median follow up: 5.3 years) [18]. In 
this study, patients who received ipilimumab $(n=475)$ had significantly higher rates of 5-year recurrence-free survival ( $40.8 \%$ versus $30.3 \%, \mathrm{p}<0.001), 5$-year distant metastasis-free survival $(48.3$ versus $38.9, \mathrm{p}=0.002)$ and 5 -year overall survival $(65.4 \%$ versus $54.4 \%$; HR for death $=0.72$, $\mathrm{p}=0.001)$ than patients who were prescribed the placebo $(\mathrm{n}=476)$.

A further randomized, double-blind, phase 3 trial evaluated the efficacy of nivolumab $(n=453)$ versus ipilimumab $(\mathrm{n}=453)$ in patients with stage IIIB, IIIC or IV melanoma who had undergone complete regional lymphadenectomy or resection [19]. Recurrence-free survival at 1 year was significantly higher in patients treated with nivolumab than in those treated with ipilimumab (70.5\% versus $60.8 \%$ ), and the risk of recurrence or death was lower $(34.0 \%$ versus $45.5 \%$ respectively, $\mathrm{HR}=0.65,97.56 \% \mathrm{CI}, 0.51-0.83 ; \mathrm{p}<0.001$ ).

In addition, as $B R A F$ mutations are found in approximately $40 \%$ of melanomas [20], a double-blind placebocontrolled phase 3 trial (COMBI-AD: $\mathrm{N}=870$, median follow up: 2.8 years) investigated the efficacy of a combination therapy with the BRAF inhibitor dabrafenib and the MEK inhibitor trametinib. This treatment improved the estimated 3 -year rate of relapse-free survival compared with a placebo (58\% versus $39 \%$; HR for relapse or death $=0.47 ; 95 \% \mathrm{CI}$, $0.39-0.58 ; \mathrm{p}<0.001)$ in patients with stage III melanoma with BRAF V600E or V600K mutations who had undergone CLND [20].

The role of checkpoint inhibitors and additional targeted therapies as adjuvant treatments is an active field of investigation for patients with stage III melanoma. Further studies are needed to determine which types of adjuvant therapies are the most effective for stage III melanoma and to identify which patients would benefit from and should be selected to receive these therapies to improve outcomes for this group of patients.

\section{Conclusion}

Overall, the benefits of SNLB have been unequivocally demonstrated and the rationale remains strong for using this procedure in patients with cutaneous melanoma. SNLB provides the most significant prognostic marker for cutaneous melanoma and will probably remain the standard of care in the management of patients with intermediate-thickness and thick cutaneous melanoma for years to come. Evidence strongly suggests that any survival benefit from node interventions in patients with nodal metastases most likely derives from SLNB. In addition, most patients with nodal metastases achieve regional lymph node control through SLNB alone. In light of the considerable advances in melanoma treatment, SLNB also provides important and accurate information about melanoma staging, which is critical for the management of melanoma patients and necessary for making therapeutic decisions concerning adjuvant therapies and for the design of future clinical trials.

Acknowledgements The author thanks Emma Pilling, Ph.D, Marianne Pons, Ph.D, Marielle Romet, Ph.D (Synergy Pharm) who provided medical writing assistance funded by Laboratoires dermatologiques Avène.

\section{Compliance with Ethical Standards}

Funding Medical writing assistance was funded by Laboratoires dermatologiques Avène. Abel Gonzalez received funds from Laboratoires dermatologiques Avène for presenting at the conference.

Conflict of interest Abel Gonzalez declares that he has no conflicts of interest that might be relevant to the contents of this manuscript.

Disclosure statement This article is published as part of a journal supplement wholly funded by Laboratoires dermatologiques Avène.

Open Access This article is distributed under the terms of the Creative Commons Attribution-NonCommercial 4.0 International License (http://creativecommons.org/licenses/by-nc/4.0/), which permits any noncommercial use, distribution, and reproduction in any medium, provided you give appropriate credit to the original author(s) and the source, provide a link to the Creative Commons license, and indicate if changes were made.

\section{References}

1. Morton DL, Thompson JF, Cochran AJ, Mozzillo N, Nieweg OE, Roses DF, et al. Final trial report of sentinel-node biopsy versus nodal observation in melanoma. N Engl J Med. 2014;370(7):599-609.

2. Snow HL. Melanotic cancerous disease. Lancet. 1892;2:872.

3. Balch CM, Soong SJ, Bartolucci AA, Urist MM, Karakousis CP, Smith TJ, et al. Efficacy of an elective regional lymph node dissection of 1 to $4 \mathrm{~mm}$ thick melanomas for patients 60 years of age and younger. Ann Surg. 1996;224(3):255-63 (discussion 63-6).

4. Cascinelli N, Morabito A, Santinami M, MacKie RM, Belli F. Immediate or delayed dissection of regional nodes in patients with melanoma of the trunk: a randomised trial. WHO Melanoma Programme. Lancet. 1998;351(9105):793-6.

5. Veronesi U, Adamus J, Bandiera DC, Brennhovd O, Caceres E, Cascinelli N, et al. Delayed regional lymph node dissection in stage I melanoma of the skin of the lower extremities. Cancer. 1982;49(11):2420-30.

6. Sim FH, Taylor WF, Pritchard DJ, Soule EH. Lymphadenectomy in the management of stage I malignant melanoma: a prospective randomized study. Mayo Clin Proc. 1986;61(9):697-705.

7. Morton DL, Wen DR, Wong JH, Economou JS, Cagle LA, Storm FK, et al. Technical details of intraoperative lymphatic mapping for early stage melanoma. Arch Surg. 1992;127(4):392-9.

8. Holmes EC, Moseley HS, Morton DL, Clark W, Robinson D, Urist MM. A rational approach to the surgical management of melanoma. Ann Surg. 1977;186(4):481-90.

9. Morton DL, Chan AD. The concept of sentinel node localization: how it started. Semin Nucl Med. 2000;30(1):4-10. 
10. Balch CM, Buzaid AC, Soong SJ, Atkins MB, Cascinelli N, Coit DG, et al. Final version of the American Joint Committee on Cancer staging system for cutaneous melanoma. J Clin Oncol. 2001;19(16):3635-48.

11. Morton DL, Hoon DS, Cochran AJ, Turner RR, Essner R, Takeuchi H, et al. Lymphatic mapping and sentinel lymphadenectomy for early-stage melanoma: therapeutic utility and implications of nodal microanatomy and molecular staging for improving the accuracy of detection of nodal micrometastases. Ann Surg. 2003;238(4):538-49 (discussion 49-50).

12. Starz H, Balda BR, Kramer KU, Buchels H, Wang H. A micromorphometry-based concept for routine classification of sentinel lymph node metastases and its clinical relevance for patients with melanoma. Cancer. 2001;91(11):2110-21.

13. Dewar DJ, Newell B, Green MA, Topping AP, Powell BW, Cook MG. The microanatomic location of metastatic melanoma in sentinel lymph nodes predicts nonsentinel lymph node involvement. J Clin Oncol. 2004;22(16):3345-9.

14. van Akkooi AC, Nowecki ZI, Voit C, Schafer-Hesterberg G, Michej W, de Wilt JH, et al. Sentinel node tumor burden according to the Rotterdam criteria is the most important prognostic factor for survival in melanoma patients: a multicenter study in 388 patients with positive sentinel nodes. Ann Surg. 2008;248(6):949-55.
15. Reeves ME, Delgado R, Busam KJ, Brady MS, Coit DG. Prediction of nonsentinel lymph node status in melanoma. Ann Surg Oncol. 2003;10(1):27-31.

16. Leiter U, Stadler R, Mauch C, Hohenberger W, Brockmeyer N, Berking $\mathrm{C}$, et al. Complete lymph node dissection versus no dissection in patients with sentinel lymph node biopsy positive melanoma (DeCOG-SLT): a multicentre, randomised, phase 3 trial. Lancet Oncol. 2016;17(6):757-67.

17. Faries MB, Thompson JF, Cochran AJ, Andtbacka RH, Mozzillo N, Zager JS, et al. Completion dissection or observation for sentinel-node metastasis in melanoma. N Engl J Med. 2017;376(23):2211-22.

18. Eggermont AM, Chiarion-Sileni V, Grob JJ, Dummer R, Wolchok JD, Schmidt H, et al. Prolonged survival in stage III melanoma with ipilimumab adjuvant therapy. N Engl J Med. 2016;375(19):1845-55.

19. Weber J, Mandala M, Del Vecchio M, Gogas HJ, Arance $\mathrm{AM}$, Cowey $\mathrm{CL}$, et al. Adjuvant nivolumab versus ipilimumab in resected stage III or IV melanoma. N Engl J Med. 2017;377(19):1824-35.

20. Long GV, Hauschild A, Santinami M, Atkinson V, Mandalà $\mathrm{M}$, Chiarion-Sileni V, et al. Adjuvant dabrafenib plus trametinib in stage III BRAF-mutated melanoma. N Engl J Med. 2017;377(19):1813-23. 